\title{
Functional clinical typology of the foot and kinematic gait parameters
}

\author{
Jitka Marenčákovái,*, Zdeněk Svoboda ${ }^{2}$, Ivan Vařeka ${ }^{2,3}$, and František Zahálka ${ }^{1}$ \\ ${ }^{I}$ Faculty of Physical Education and Sport, Charles University, Prague, Czech Republic; ${ }^{2}$ Faculty of Physical Culture, Palacký \\ University Olomouc, Czech Republic; and ${ }^{3}$ Faculty of Medicine, Charles University, Hradec Králové, Czech Republic
}

Copyright: (C) 2016 J. Marenčáková et al. This is an open access article licensed under the Creative Commons Attribution License (http://creativecommons.org/licenses/by/4.0/).

\begin{abstract}
Background: The foot plays a key role in a standing posture, walking and running performance. Changes in its structure or function may alter upper segments of kinematic chain which can lead to formation of musculoskeletal disorders. Although functional clinical typology provides a complex view of foot kinesiology there is a lack of knowledge and evidence about influences of different foot types on human gait. Objective: The aim of the study was to analyse differences of kinematic gait parameters of lower extremity joints and pelvis between functional clinical foot types in healthy young men. Methods: Three-dimensional kinematic analysis by the Vicon Motion Capture MX System device in synchronization with 2 Kistler force platforms was used to obtain kinematic data from 18 healthy men (mean age $23.2 \pm 1.9$ years). The functional clinical foot type was clinically examined and sorted into 3 basic foot type groups - forefoot varus (FFvar), rearfoot varus (RFvar) and forefoot valgus (FFvalg). Peak angular values and range of an angular displacement in all of three movement planes were analysed for pelvis, hip, knee and ankle joint. For statistical analysis of kinematic gait parameters differences between foot types Mann Whitney $U$ test at a statistical significance level $p<.05$ and Cohen's coefficient $d$ for effect size were used. Results: This study showed that functional clinical foot type can affect kinematic parameters of gait in the joints of the lower limb and pelvis. Significant differences were presented in the FFvar in comparison with other two foot type groups with middle and high size of effect. The most alterations were observed in pelvis area and in a sagittal plane of movement. Nevertheless, significant differences between FFvalg and RFvar foot types were not noticed. Conclusions: Functional clinical foot typology provides one of the possible methods to describe foot structure and function. Our results showed that foot type could alter gait. Forefoot varus foot type has more significant influence on kinematic gait parameters then other foot types. These effects should be considered by clinicians during examination of musculoskeletal system disorders especially in lower extremities.
\end{abstract}

Keywords: foot, functional typology, gait cycle, kinesiology, 3D kinematic analysis

\section{Introduction}

The human foot is the only segment of the lower limb that provides direct contact with the ground during standing, walking and running (Tiberio, 1988). Its complex anatomical structure is designed to ensure both the function of static support and simultaneously dynamic locomotor function (Dylevský, 2009). That puts claim at a certain phase of gait cycle to be flexible enough for absorbing the shock during initial contact and adapt to uneven terrain and at a different phase conversely be sufficiently rigid when one creates an

\footnotetext{
* Address for correspondence: Jitka Marenčáková, Sport Research Centre, Faculty of Physical Education and Sport, Charles University, José Martího 31, 16252 Praha 6, Czech Republic. E-mail: marencakova.jitka@seznam.cz
}

impulse to move forward (Scott, Menz, \& Newcombe, 2007). It is also involved in managing and maintaining stability and balance (Cote, Brunet, Gansneder, \& Shultz, 2005; Lewit \& Lepšíková, 2008).

Foot typology is a clinical concept that targets to simplify the anatomical complexities of the human foot (Hillstrom et al., 2013). Currently there are several methods for determining foot type but most of them evaluate foot morphology in its static position. Although no universal consensus exists for the ideal method of the foot type classification it is possible to divide them into the basic categories - visual non-quantitative clinical inspection (e.g. arch height, navicular prominences, position of rearfoot), anthropometric values (e.g. valgus index, navicular drop, rearfoot angle), footprint parameters (e.g. arch index) and radiographic evaluation (e.g. calcaneal inclination 
angle) (Billis, Katsakiori, Kapodistrias, \& Kapreli, 2007; Buldt et al., 2013; Hillstrom et al., 2013; Ozer, 2012; Razeghi \& Batt, 2002).

In the 60's of 20th century Merton Root came up with a new, so-called functional clinical typology of the foot depending on a position of rearfoot relative to shin and forefoot relative to rearfoot (Tiberio, 1988). He described the foot primarily as a dynamic complex and took into account the position of the loaded and unloaded foot. He introduced the clinical term neutral position of the subtalar joint (NPSJ) which was progressively modified into a position where subtalar joint (SJ) is neither prone nor supine (Root, Orien, Weed, \& Hughes, 1971). Root, his colleagues and followers described four basic types called intrinsic foot deformities - forefoot varus, forefoot valgus and rearfoot varus, and their sub-types and defined the normal foot (Buchanan \& Davis, 2005). Root's typology has brought a new insight into the kinesiology, pathokinesiology and biomechanics of the foot and it became the basis of compensatory, not a corrective, orthoses concept using struts and wedges, which aims to prevent self-compensation mechanisms of the lower limbs segments with their negative consequences (Eslami, Tanaka, Hinse, Farahpour, \& Allard, 2006). On the other hand, it is still criticized for low reliability in determining foot type and there is still no general definition of neutral position of subtalar joint (Cornwall et al., 2004; Lee, 2001; Razeghi \& Batt, 2002). However, despite questioning Root's definitions and methods for determining NPSJ, it is still the gold standard for a reference position of the foot and it is used for the design and a production of high efficiency functional braces (Harradine, Bevan, \& Carter, 2006; Miller \& McGuire, 2000).

In all types of the foot there are compensatory mechanisms that are required to achieve the necessary contact of the foot during the stance phases of the gait cycle. Joints of the foot work with the other joints of the lower limb within the open and closed kinematic chains and it is known that the associated movements exist (Betsch et al., 2011). Inadequate settings of the motion segments of the lower limbs thus may gradually lead to an asymmetric load musculoskeletal structures and thereby cause pathology of the musculoskeletal system (Billis et al., 2007; Curran \& Dananberg, 2005; Hsi, 2016; Vařeka, 2004), e.g. deformities of the toes (Vařeka \& Vařeková, 2009), patellofemoral pain syndrome (Barton, Levinger, Crossley, Webster, \& Menz, 2012) or even pain in the lumbar spine (Michaud, 1997).

Variability in kinematics of the foot is high to normal as was evaluated on dynamic cadavers (Nester, 2009). We hypothesized that each foot type can differently influence the kinematic chain of the lower extremity and pelvis. Thus the aim of this study was to clarify whether there are differences in the kinematic parameters of the joints of the lower extremity and pelvis during gait cycle between different functional clinical foot types.

\section{Methods}

\section{Participants}

Fifty men, students of the Faculty of Physical Culture, Palacký University Olomouc, volunteered to take a part in the study but only 18 participants (mean age $23.2 \pm 1.9$ years, body mass $77.8 \pm 8.1 \mathrm{~kg}$, and body height $181.0 \pm 5.1 \mathrm{~cm}$ ) had completed all procedures. Each participant filled out an entry questionnaire about his health status, so we excluded those individuals who have had an injury of lower limb, pelvis or spine and persons with sensory nerve damage or mental illnesses. Prior to the study, participants were fully informed about the study design and they signed an informed consent form. The project was approved by the Ethics Committee of the Faculty of Physical Culture, Palacký University Olomouc.

\section{Measurement}

The three-dimensional gait analysis was supported by the Vicon Motion Capture MX System (Vicon Motion Systems, Oxford, United Kingdom). This system was based on 7 infrared cameras Vicon MX (T10, 1 megapixel) connected to the MX Ultranet controlling hardware. Kinetic data were collected using 2 Kistler force platforms $60 \times 40 \mathrm{~cm}$ (type 9286AA, Kistler Instrumente, Winterthur, Switzerland). This equipment was connected to the Vicon MX Control in order to guarantee the synchronization of Vicon MX Ultranet with the Vicon Motion Capture System.

Force platforms were placed between the wooden floorboards so that their edges weren't above the floor and did not therefore affect the natural gait cycle. Kinematic and kinetic data were recorded at $200 \mathrm{~Hz}$ frequency. The placement of the 16 spherical reflective markers (14 $\mathrm{mm}$ diameter) on the subject's anatomical landmarks was in accordance with PlugInGait model (Vicon Motion Systems, Oxford, United Kingdom).

The calibration procedure was developed in accordance with the Vicon technical specifications. For static calibration we used calibration L-frame and for dynamic calibration we used calibration wand. Cameras placement was in accordance with the manufacturer's instructions. All kinematic data were filtered by Woltring filtering routine $(10 \mathrm{~Hz})$. We evaluated peak values and range of an angular displacement in 
segments of the lower extremities and pelvis during normal gait cycle.

\section{Procedure}

Before kinematic gait analysis every participant followed an anthropometric measurements using for individualization of the software model (establishing centres of joints, etc.). One examiner measured height, weight and functional leg length (distance between the medial malleolus and the anterior superior iliac spine). Furthermore, the width of the shoulder, elbow width, the width of the wrist, the distance between the dorsal and palmar side of the hand, the distance between the centre of the shoulder joint and acromion, the width of the knee and the ankle width were measured.

Prior to each test, laboratory space, cameras, force plates and the entire system were calibrated as recommended. Participants walked barefoot and only in their underwear. Firstly, we put 16 spherical reflective markers on the anatomical points of participant's body on the both sides - spina iliaca anterior superior, spina iliaca posterior superior, trochanter major femoris, epicondylus lateralis femoris, tuberositas tibiae, malleolus lateralis fibulae, os calcaneus, articulatio metatarsophalangeae I. Then we measured the person in a quiet standing position to allow the software to recognize and name designated points. The participant was then asked to walk for training several times across the $8 \mathrm{~m}$ long path with his natural speed and to walk through measuring power plates so that his heel and sole of one lower limb are put on the one measurement platform and the another lower limb in the same way on the second platform. After the training session at least six valid attempts were recorded. To eliminate the influence of speed on gait cycle we selected only those session when the participant's walking speed was in the range $1.38-1.52 \mathrm{~m} \cdot \mathrm{s}^{-1}$. This range was determined experimentally before carrying out the research by a group of 10 men in the same age walking by their natural walking speed.

After obtaining data from the kinematic analysis we continued with an examination of the functional clinical foot type. The participants were individually examined by one skilled physician. Determining functional clinical foot type was done in a prone position with both legs hanging over the edge of a bed. The participant bent one leg on the side and placed the heel of the leg to the popliteal area of investigated lower limb to ensure its neutral position. Then the examiner visually evaluated the rearfoot position relative to the distal third of the axis of shank and the forefoot plane position relative to the rearfoot plane of straightened leg (Valmassy, 1996). Three functional clinical foot types were determined forefoot varus (FFvar), rearfoot varus
(RFvar) and forefoot valgus (FFvalg). He compared the findings in each foot when unloaded and loaded during the subject's standing position and determined the compensated and uncompensated, flexible and rigid subtypes. Due to the small research sample we did not take subtypes into account in our study. To simplify the classification we did not establish the types supinated forefoot and plantar flexed first ray. They were regarded as forefoot varus (for supinated forefoot) and as forefoot valgus (for plantar flexed first ray) as it was repeatedly published earlier (Valmassy, 1996; Vařeka \& Vařeková, 2008).

\section{Statistical methods}

Obtained data were analysed with the software Vicon Nexus and Vicon Polygon and they were sorted in the Microsoft Office Excel (Version 2007; Microsoft, Redmond, WA, USA). For statistical analysis we used the Statistica program (Version 10; StatSoft, Tulsa, OK, USA). Since data were not normally distributed, we used the non-parametric Mann Whitney $U$ test with statistical significance determined at the level $p<.05$. For evaluation of size of effect we calculated Cohen's coefficient $d(0.20 \leq d<0.50$ small effect; $0.50 \leq d<0.80$ medium effect; $d \geq 0.80$ high effect).

\section{Results}

Using 3D kinematic analysis we observed angular kinematic parameters of the lower extremity joints and pelvis in the sagittal, frontal and transversal plane during gait cycle among functional clinical foot types (Table 1, Figure 1).

We found higher first plantar flexion of ankle during loading response phase in FFvalg compare to FFvar group ( $p=.01 ; d=2.42$ ). Parameter external rotation of the knee during mid-swing phase was higher in RFvar compared to FFvar group $(p=.01 ; d=0.94)$. Furthermore, there was higher range of rotation of hip (respectively femur) in FFvar compared to RFvar group ( $p=.03 ; d=0.89$ ) and higher adduction of hip during loading response phase for RFvar compared to FFvar group $(p=.01 ; d=1.09)$. Parameter anteversion peak of pelvis in terminal stance phase was higher in FFvar compared to FFvalg group ( $p=.04 ; d=1.20)$. Parameter retroversion peak of pelvis during loading response to mid-swing phase was higher in FFvar compared to FFvalg group ( $p=.03 ; d=1.35)$ and compared to RFvar group $(p=.01 ; d=1.54)$. Internal rotation of pelvis during initial contact phase and terminal swing phase was higher in FFvar compared to FFvalg group $(p=.04 ; d=0.88)$. 
Table 1

Kinematic gait parameters (peak values and range of motion, given in degrees) of lower limb joints and pelvis and their differences between functional clinical foot types

\begin{tabular}{|c|c|c|c|c|c|c|c|c|}
\hline \multirow[b]{2}{*}{$\begin{array}{l}\text { Joint/ } \\
\text { Segment }\end{array}$} & \multirow[b]{2}{*}{ Plane } & \multirow[b]{2}{*}{ Variable } & \multirow{2}{*}{$\begin{array}{l}\text { Forefoot } \\
\text { valgus } \\
(n=5)\end{array}$} & \multirow{2}{*}{$\begin{array}{c}\text { Rearfoot } \\
\text { valgus } \\
(n=20)\end{array}$} & \multirow{2}{*}{$\begin{array}{c}\text { Rearfoot } \\
\text { valgus } \\
(n=11)\end{array}$} & \multicolumn{3}{|c|}{ Significance level } \\
\hline & & & & & & $\begin{array}{l}\text { FFvalg } \\
\text { vs FFvar }\end{array}$ & $\begin{array}{l}\text { FFvalg } \\
\text { vs RFvar }\end{array}$ & $\begin{array}{c}\text { FFvar } \\
\text { vs RFvar }\end{array}$ \\
\hline \multirow[t]{7}{*}{ Ankle } & Sagittal & First plantar flexion & $14.5 \pm 4.9$ & $6.5 \pm 2.9$ & $10.3 \pm 5.3$ & .01 & .18 & .07 \\
\hline & & Dorsal flexion & $9.9 \pm 2.4$ & $8.9 \pm 3.7$ & $8.4 \pm 2.6$ & .37 & .27 & .86 \\
\hline & & Second plantar flexion & $-21.6 \pm 2.6$ & $-17.3 \pm 7.2$ & $-22.8 \pm 6.5$ & .13 & $>.99$ & .06 \\
\hline & & ROM & $32.1 \pm 1.8$ & $26.4 \pm 7.1$ & $31.2 \pm 7.5$ & .08 & .74 & .15 \\
\hline & Transversal & Internal rotation & $-0.6 \pm 8.3$ & $4.5 \pm 18.2$ & $4.1 \pm 7.1$ & .41 & .51 & .76 \\
\hline & & External rotation & $-21.7 \pm 11.5$ & $-25.7 \pm 12.6$ & $-18.9 \pm 11.0$ & .37 & .91 & .28 \\
\hline & & ROM & $21.1 \pm 4.5$ & $30.1 \pm 14.9$ & $23.0 \pm 11.2$ & .07 & .32 & .56 \\
\hline \multirow[t]{11}{*}{ Knee } & Sagittal & First flexion & $40.0 \pm 8.1$ & $40.8 \pm 5.5$ & $43.7 \pm 10.1$ & .67 & .66 & .64 \\
\hline & & First extension & $6.3 \pm 3.8$ & $7.6 \pm 4.7$ & $5.1 \pm 3.9$ & .67 & .51 & .11 \\
\hline & & Second flexion & $63.0 \pm 5.1$ & $62.4 \pm 7.8$ & $62.8 \pm 8.6$ & .97 & .83 & .89 \\
\hline & & Second extension & $1.2 \pm 4.1$ & $2.3 \pm 7.6$ & $-0.2 \pm 7.8$ & .97 & .32 & .26 \\
\hline & & $\mathrm{ROM}$ & $63.1 \pm 7.1$ & $61.3 \pm 4.3$ & $65.7 \pm 8.4$ & .92 & .51 & .10 \\
\hline & Frontal & Adduction & $7.7 \pm 8.6$ & $2.7 \pm 4.4$ & $1.9 \pm 3.2$ & .34 & .15 & .58 \\
\hline & & Abduction & $-12.9 \pm 13.3$ & $-14.4 \pm 10.0$ & $-18.8 \pm 9.3$ & .34 & .27 & .16 \\
\hline & & ROM & $20.6 \pm 10.6$ & $17.1 \pm 7.2$ & $20.6 \pm 9.0$ & .67 & $>.99$ & .21 \\
\hline & Transversal & Internal rotation & $22.8 \pm 7.2$ & $33.9 \pm 24.5$ & $23.7 \pm 11.3$ & .49 & .91 & .36 \\
\hline & & External rotation & $-6.9 \pm 6.1$ & $3.9 \pm 18.4$ & $-11.0 \pm 8.9$ & .19 & .66 & .01 \\
\hline & & ROM & $29.6 \pm 8.5$ & $30.0 \pm 13.3$ & $34.6 \pm 10.3$ & .67 & .32 & .15 \\
\hline \multirow[t]{9}{*}{ Hip } & Sagittal & Flexion & $24.8 \pm 1.9$ & $29.9 \pm 6.0$ & $26.1 \pm 2.8$ & .07 & .18 & .06 \\
\hline & & Extension & $-17.9 \pm 4.2$ & $-11.6 \pm 6.0$ & $-13.0 \pm 5.3$ & .06 & .07 & .43 \\
\hline & & ROM & $42.7 \pm 4.5$ & $41.5 \pm 5.2$ & $39.1 \pm 6.2$ & .67 & .44 & .36 \\
\hline & Frontal & Adduction & $6.2 \pm 2.2$ & $5.0 \pm 2.3$ & $7.5 \pm 2.2$ & .30 & .27 & .01 \\
\hline & & Abduction & $-5.9 \pm 1.5$ & $-7.3 \pm 2.4$ & $-6.4 \pm 3.5$ & .24 & .74 & .58 \\
\hline & & ROM & $12.1 \pm 2.1$ & $12.4 \pm 3.7$ & $13.9 \pm 2.7$ & .97 & .18 & .16 \\
\hline & Transversal & External rotation & $10.7 \pm 6.1$ & $1.1 \pm 23.6$ & $11.8 \pm 9.7$ & .17 & .91 & .28 \\
\hline & & Internal rotation & $-11.8 \pm 4.1$ & $-15.0 \pm 19.8$ & $-11.0 \pm 4.1$ & .41 & .58 & .38 \\
\hline & & ROM & $22.6 \pm 9.8$ & $16.2 \pm 6.8$ & $22.8 \pm 8.5$ & .06 & .38 & .03 \\
\hline \multirow[t]{9}{*}{ Pelvis } & Sagittal & Anteversion & $8.9 \pm 3.2$ & $12.5 \pm 2.9$ & $8.7 \pm 6.5$ & .04 & .91 & .16 \\
\hline & & Retroversion & $7.4 \pm 3.7$ & $11.6 \pm 2.9$ & $5.6 \pm 5.2$ & .03 & .51 & .01 \\
\hline & & ROM & $2.9 \pm 0.9$ & $3.5 \pm 0.8$ & $3.1 \pm 1.3$ & .19 & $>.99$ & .19 \\
\hline & Frontal & Obliquity up & $4.5 \pm 1.1$ & $5.4 \pm 1.6$ & $4.9 \pm 1.2$ & .37 & .58 & .48 \\
\hline & & Obliquity down & $-4.6 \pm 1.2$ & $-5.2 \pm 1.7$ & $-5.1 \pm 2.1$ & .49 & .74 & .76 \\
\hline & & $\mathrm{ROM}$ & $9.1 \pm 1.8$ & $10.6 \pm 2.7$ & $10 \pm 2.6$ & .24 & .83 & .67 \\
\hline & Transversal & Internal rotation & $5.6 \pm 1.6$ & $7.8 \pm 2.6$ & $7.0 \pm 3.9$ & .04 & .18 & $>.99$ \\
\hline & & External rotation & $-5.3 \pm 2.5$ & $-6.4 \pm 3.0$ & $-6.8 \pm 4.1$ & .45 & .27 & .40 \\
\hline & & ROM & $10.9 \pm 3.8$ & $14.1 \pm 4.2$ & $13.8 \pm 5$ & .13 & .22 & .95 \\
\hline
\end{tabular}

Note. $\quad$ FFvalg = forefoot valgus; FFvar = forefoot varus; RFvar = rearfoot varus; ROM = range of motion. Statistically significant differences are in boldface.

\section{Discussion}

Based on the results we can say that the functional clinical type of foot tends to affect the kinematic parameters of the lower extremity joints and pelvis during walking. Most differences were found for the forefoot varus and most in the transverse plane, where rotations are mainly present.

Forefoot varus (FFvar) was the most strongly represented type in our sample $(55.6 \%)$. It is characterized 

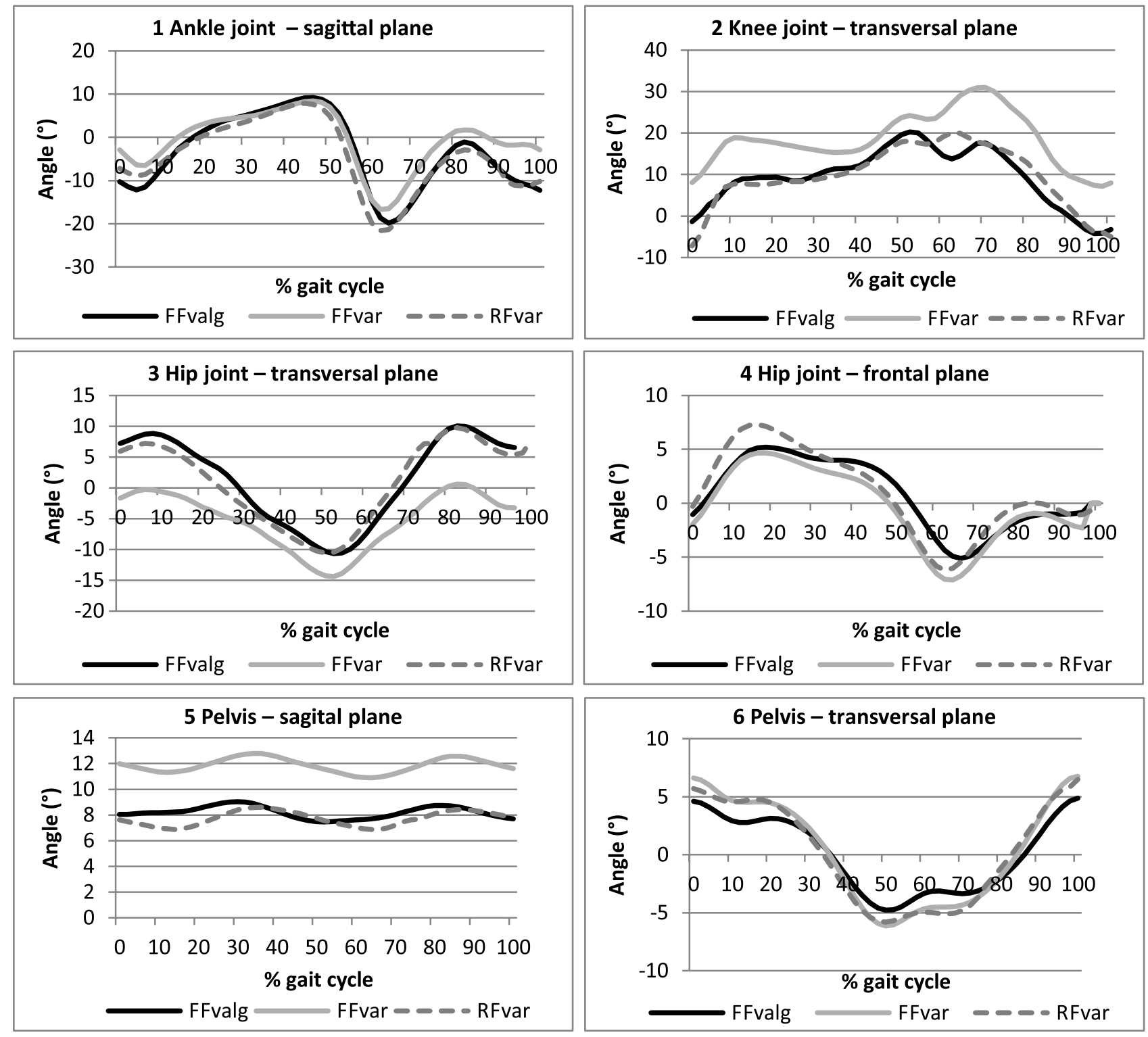

Figure 1. Graphical representation of mean values of kinematic parameters of the lower extremity joints and pelvis during walking in different foot types. FFvalg = forefoot valgus; FFvar = forefoot varus; RFvar = rearfoot varus.

by the forefoot in relative supination to the rearfoot (Tiberio, 1988). The main strategy of compensatory mechanisms for FFvar lies in the extended or excessive pronation during mid and final stance phase with compensated forefoot varus. For the uncompensated variant of FFvar the medial midfoot portion remains without contact with the ground and thereby, the concentration of weight at the lateral edge of the sole lasts until the heel strike (Vařeka \& Vařeková, 1999). Our study did not confirm these combined movements directly because we did not find significant changes in kinematic parameters in ankle joint (AJ) in the transverse plane, but we found differences in the upper segments, at the level of the pelvis and hip joint. According to theoretical knowledge of kinematic chains, rearfoot pronation within a closed kinematic chain causes internal rotation of tibia and fibula (Rodrigues, Chang, TenBroek, van Emmerik, \& Hamill, 2015), followed by flexion of the knee joint, the femur internally rotates and thereby increases pelvic anteversion (Kapandji, 1987; Souza, Draper, Fredericson, \& Powers, 2010; Tiberio, 1988). This was confirmed in our study. In the FFvar group we found higher anteversion and retroversion peak. Pelvis was in a mean value $12.5^{\circ}$ $\left(\mathrm{SD} 2.9^{\circ}\right)$ of anteversion in its basic position which is higher than the indicated norm. This increased initial anteversion of the pelvis may be caused by mechanisms in FFvar type during walking which can lead to greater and faster rearfoot hyperpronation, which reduces supination at the end of the swing phase and decreases 
the ability to absorb the shock during initial contact. Perry and Burnfield (2010) note if during the gait cycle more anteversion of pelvis occurs then it must occur at the same time also compensation by increasing range of flexion in hip joint, to maintain the stride length. This increased flexion of the hip in our study did not manifest, but rather the value of a hip internal rotation increased, which may be another possible compensatory mechanism to maintain the length of the step in the group FFvar.

Rearfoot varus (RFvar) is according to Vařeka and Vařeková (2009) the most frequently occurring type of foot in males. In our study, rearfoot varus was represented only in $30.6 \%$ as the second most common type of foot. RFvar is characterized by supination position of whole foot when subtalar joint (ST) is in a neutral position, specifically calcaneus in supination and the heel in varus. There are two types - rearfoot varus and tibial varus (McPoil, Knecht, \& Schuit, 1988). In our study we did not differentiate these subtypes. Also in this foot type, compensatory mechanisms may involve gait cycle adjustment as was described by authors Mooney and Campbell (2006) as "an abduction twist" which is characterized by an increased external rotation of the tips of feet while walking. This allows the outer edge of the forefoot been used as a pivot, which rapidly allows preparation during heel strike for subsequent loading of the medial forefoot in the next phase of mid-stance. This was confirmed in our study and reflected in the increased value of the parameter external rotation of knee joint. Furthermore, this group has increased the value of adduction peak of the hip joint compared to normal value and to other two groups. This chaining supports study by Barton et al. (2012), which shows a direct correlation between an increased range of rearfoot eversion and increased range of adduction of femur in healthy subjects.

Forefoot valgus (FFvalg), according to a study of McPoil et al. (1988), is considered the most common type of foot in the population, in our study, however, it occurred only in $13.9 \%$. The reason could be due to our small research sample. If the ST joint is in the neutral position, forefoot is located in pronation relative to rearfoot (Tiberio, 1988). Valmassy (1996) talked about the division into two subtypes - flexible and rigid. Flexible FFvalg has a sufficient degree of compensation by forefoot supination around the longitudinal axis of the transverse tarsal joint (TT). However, it changes the foot settings, because the pronation in the TT joint unlocks the forefoot, it becomes unstable and unfavourable for foot reflection. Michaud (1997) distinguishes A, B1 and B2 subtypes of flexible FFval. Subtype A has a large range of supination in the joint TT, which in turn leads to the hyperpronation syndrome pathology described by FFvar. Subtype B1 has enlarged range of pronation in TT joint (up to $6.0^{\circ}$ ). This is compensated by accentuated ST joint supination at the beginning of reflection till supination chronic overloading gradually occurs. Subtype B2 is manifested by hypersupination already at the end of the mid-stance, that increasing internal rotation of tibia and overloading the m. peroneus longus. Rigid form of FFvalg does not allow any compensation and foot has a high medial arch and supine heel. We have not considered these subtypes in our research due to the small research sample. On the other hand, the kinematic parameter peak plantar flexion of the ankle joint during loading response was different between forefoot valgus and forefoot varus types. The forefoot valgus group was characterized by the highest mean value of the parameter compared to the normal value (Perry \& Burnfield, 2010) and to the mean values of other types. We did not expect these changes because of theoretical knowledge. Compensatory mechanisms more often occur in the frontal and transverse plane, especially at the level of ankle respectively subtalar joint (Daniels, Smith, \& Ross, 1996). We used three-pointed model of foot in our research hence we evaluated complex movement of ankle and foot joints as a unit. AJ movements do not happen purely in the sagittal plane. Although the main function of ankle joint is plantar and dorsal flexion, due to the course of the joint axis which is directed obliquely "through the posterolateral aspect of the calcaneus and anteriorly over the head of the first metatarsal" (Kirby, 2000 , p. 3), the movement of the small scale takes place also in the transversal plane. Plantar flexion therefore also contains adduction and inversion component of movement and conversely dorsal flexion contains abduction and eversion movement component (Neumann, 2010) which could explain our results. We can see the tendency to foot specific chaining patterns at least for FFvar type but our study did not show that yet significantly because of study limitations as were a small research sample and no distinguishing between foot subtypes.

\section{Conclusions}

Functional clinical foot typology provides one of the possible methods to describe foot complex structure and dynamic function. Forefoot varus has more significant influence on these gait parameters in comparison with other foot types. Nevertheless, we did not find significant difference between forefoot valgus and rearfoot varus. Further research is needed for evaluation of foot type specific chaining pattern to better understand pathokinesiology and possible causes of 
musculoskeletal disorders and to establish optimal preventive proceedings especially among forefoot varus individuals.

\section{Acknowledgments}

Research wassupported bygrants IGA_FTK_2015_006, SVV FTVS 260236 and PRVOUK 038.

\section{Conflict of interest}

There were no conflicts of interest.

\section{References}

Barton, C. J., Levinger, P., Crossley, K. M., Webster, K. E., \& Menz, H. B. (2012). The relationship between rearfoot, tibial and hip kinematics in individuals with patellofemoral pain syndrome. Clinical Biomechanics, 27, 702-705.

Betsch, M., Schneppendahl, J., Dor, L., Jungbluth, P., Grassmann, J. P., Windolf, J., ... Wild, M. (2011). Influence of foot positions on the spine and pelvis. Arthritis Care \& Research, 63, 1758-1765.

Billis, E., Katsakiori, E., Kapodistrias, C., \& Kapreli, E. (2007). Assessment of foot posture: Correlation between different clinical techniques. Foot, 17, 65-72.

Buchanan, K. R., \& Davis, I. (2005). The relationship between forefoot, midfoot and rearfoot static alignment in pain-free individuals. Journal of Orthopaedic \& Sports Physical Therapy, 35, 559-566.

Buldt, A. K., Murley, G. S., Butterworth, P., Levinger, P., Menz, H. B., \& Landorf, K. B. (2013). The relationship between foot posture and lower limb kinematics during walking: A systematic review. Gait \& Posture, 38, 363-372.

Cornwall, M. E., McPoil, T. G., Fishco, W. D., Hunt, L., Lane, C., \& O’Donnell, D. (2004). Reliability of visual measurement of forefoot alignment. Foot \& Ankle International, 25, 745-748.

Cote, K. P., Brunet, M. E., Gansneder, B. M., \& Shultz, S. J. (2005). Effects of pronated and supinated foot postures on static and dynamic postural stability. Journal of Athletic Training, 40, 41-46.

Curran, S. A., \& Dananberg, H. J. (2005). Future of gait analysis. A podiatric medical perspective. Journal of the American Podiatric Medical Association, 95, 130-142.

Daniels, T. L., Smith, J. W., \& Ross, T. J. (1996). Varus malalignment of the talar neck. Its effect on the position of the foot and on subtalar motion. Journal of Bone \& Joint Surgery - Series A, 78, 1559-1567.

Dylevský, I. (2009). Speciální kineziologie [Special kinesiology]. Prague, Czech Republic: Grada Publishing.

Eslami, M., Tanaka, C., Hinse, S., Farahpour, N., \& Allard, P. (2006). Effect of foot wedge positions on lower-limb joints, pelvis and trunk angle variability during single-limb stance. Foot, 16, 208-213.
Harradine, P., Bevan, L., \& Carter, N. (2006). An overview of podiatric biomechanics theory and its relation to selected gait dysfunction. Physiotherapy, 92, 122-127.

Hillstrom, H. J., Song, J., Kraszewski, A. P., Hafer, J. F., Mootanah, R., Dufour, A. B., ... Deland, J. T. (2013). Foot type biomechanics part 1: Structure and function of the asymptomatic foot. Gait \& Posture, 37, 445-451.

Hsi, W. L. (2016). Analysis of medial deviation of center of pressure after initial heel contact in forefoot varus. Journal of the Formosan Medical Association, 115, 203-209.

Kapandji, I. A. (1987). The physiology of the joints: Annotated diagrams of the mechanics of the human joints. Volume 2: Lower limb (5th ed.). London, United Kingdom: Churchill Livingstone.

Kirby, K. A. (2000). Biomechanics of the normal and abnormal foot. Journal of the American Podiatric Medical Association, 90, 1-5.

Lee, W. E. (2001). Podiatric biomechanics. An historical appraisal and discussion of the Root model as a clinical system of approach in the present context of theoretical uncertainty. International Journal of Podiatric Biomechanics, 18, 555-684.

Lewit, K., \& Lepšíková, M. (2008). Chodidlo - významná část stabilizačního systému [Foot - a significant part of the stabilization system]. Rehabilitace a fyzikální lékařství, 3, 99-104.

McPoil, T. G., Knecht, H. G., \& Schuit, D. (1988). A survey of foot types in normal females between the ages of 18 and 30 years. Journal of Orthopaedic and Sports Physical Therapy, 9, 406-409.

Michaud, T. C. (1997). Foot orthoses and other forms of conservative foot care. Newton, MA: Thomas C. Michaud.

Miller, M., \& McGuire, J. (2000). Literature reveals no consensus on subtalar neutral. Biomechanics, 7, 63-74.

Mooney, J., \& Campbell, R. (2006). General foot disorders. In D. Lorimer, G. French, M. O’Donnell, J. Gordon Burrow, \& B. Wall (Eds.), Neale's disorders of the foot (7th ed., pp. 89-164). London, United Kingdom: Churchill Livingstone.

Neumann, D. A. (2010). Kinesiology of the musculoskeletal system. Foundations for rehabilitation (2nd ed.). Maryland Heights, MO: Mosby.

Nester, C. J. (2009). Lessons from dynamic cadaver and invasive bone pin studies: Do we know how the foot really moves during gait? Journal of Foot and Ankle Research, 2, 18.

Ozer, C. M. (2012). Evaluation of the sole morphology of professional football players. International Sport \& Medicine Journal, 13, 8-17.

Perry, J., \& Burnfield, J. M. (2010). Gait analysis. Normal and pathological function (2nd ed.). Thorofare, NJ: SLACK Incorporated.

Razeghi, M., \& Batt, M. E. (2002). Foot type classification: A critical review of current methods. Gait \& Posture, 15, 282-291.

Rodrigues, P., Chang, R., TenBroek, T., van Emmerik, R., \& Hamill, J. (2015). Evaluating the coupling between foot pronation and tibial internal rotation continuously using vector coding. Journal of Applied Biomechanics, 31, 88-94. 
Root, M. L., Orien, W. P., Weed, J. H., \& Hughes, R. J. (1971). Biomechanical evaluation of the foot. Los Angeles, CA: Clinical Biomechanics Corporation.

Scott, G., Menz, H. B., \& Newcombe, L. (2007). Age-related differences in foot structure and function. Gait \& Posture, 26, 68-75.

Souza, R. B., Draper, C. E., Fredericson, M., \& Powers, C. M. (2010). Femur rotation and patellofemoral joint kinematics: A weight-bearing magnetic resonance imaging analysis. Journal of Orthopaedic \& Sports Physical Therapy, 40, 277-285.

Tiberio, D. (1988). Pathomechanics of structural foot deformities. Physical Therapy, 68, 1840-1849.
Valmassy, R. L. (Ed.). (1996). Clinical biomechanics of the lower extremities. St. Louis, MO: Mosby.

Vařeka, I. (2004). Pronace/everze v subtalárním kloubu vyvolaná flexí v kolenním kloubu $\mathrm{v}$ uzavřeném kinematickém řetězci [Pronation/eversion of the subtalar joint induced flexion of the knee in a closed kinematic chain]. Rehabilitace a fyzikální lékařství, 11, 163-168.

Vařeka, I., \& Vařeková, R. (2008). The height of the longitudinal foot arch assessed by Chippaux-Šmiřák index in the compensated foot types according to Root. Acta Universitatis Palackianae Olomucensis. Gymnica 38(1), 35-41.

Vařeka, I., \& Vařeková, R. (2009). Kineziologie nohy [Kinesiology of the foot]. Olomouc, Czech Republic: Palacký University Olomouc. 